primary meaning of the verb totella was 'to care, be concerned about'. The semantic tradition, then, may have occasionally been so powerful that it prevented the grafting of new meanings onto a word already present in the vernacular.

The author also considers the question of the relationships between translation loans with the same or similar meanings. In some cases they may have existed in parallel, perhaps even for some time, and then gradually become semantically differentiated. This occurred with the words kaatua and langeta (both meaning 'to fall'), which both took on the meaning of 'to fall in battle' under the influence of foreign models. In the 19 th century kaatua displaced its synonym. The reason may have been that the expression langeta syntiin (to lapse into sin) in religious language had become embedded so firmly in the general consciousness that the verb langeta was not readily allowed any other meaning than that of 'going astray'. In other' cases two semantically equivalent translation loans have alternated in use. For example, the meaning 'interest on capital' was originally expressed by both the words kasio and korko; but from the 17th century kasio began to displace korko, presumably because the Catholic Church condemned the taking of loans, and the word korko thus acquired pejorative connotations with 'koronkiskonta' (usury). But in the 19th century korko in its turn displaced kasvo.

At the end of the book (pp. 192-200) the author has appended a word index and an index of names. Use of the word index is facilitated by the grouping of the words by language: Finnish, other Baltic-Finnic languages, other Finno-Ugric languages, Scandinavian languages, other Germanic languages, Slavic and Baltic languages, Modern Romance languages, Latin, Greek, other languages.

Pirkku NuoliJärvi

\title{
Lappische Kasussyntax
}

RAIJA BARTENs, Inarinlapin, merilapin ja luulajanlapin kaasussyntaksi (Kasussyntax des Inari-, See- und Lulelappischen). Mémoires de la Société Finno-ougrienne 148. Helsinki $1972.180 \mathrm{~S}$.

Über dreissig Jahre sind vergangen, seit die vorhergehende umfangreiche Untersuchung über die lappische Kasussyntax erschien, Johan Beronkas "Lappische Kasusstudien". Bedenkt man die in den letzten Jahrzehnten im Bereich der Sprach- 
wissenschaften geschehene Entwicklung, so wird klar, dass alle Voraussetzungen vorhanden sind, die lappische Kasussyntax unter neuen Gesichtspunkten und mit neuen Zielen zu erörtern. Beronkas Untersuchung gehörte in den Kreis der historischen Satzlehre. Mittels der historisch-komparativen Methode sollten Herkunft und Entwicklung der syntaktischen Funktionen der wichtigsten Kasus des Lappischen erklärt werden. Die Untersuchung betraf alle lappischen Dialekte und der Vergleich zog auch die entfernteren finnisch-ugrischen Sprachen heran.

Die Untersuchung von Raija Bartens über die lappische Kasussyntax - eine akademische Abhandlung, eine Dissertation zur Erlangung des Doktorgrades - ist wiederum eine rein synchronische Darstellung der syntaktischen Funktionen der Kasus des Inari-, See- und Lulelappischen. Diese Mundarten weichen beachtlich voneinander ab. Das Inarilappische gehört zur Hauptdialektgruppe des Ostlappischen, das Lulelappische wiederum ist ein rein westlappischer Dialekt. Unter dem Begriff Seelappisch werden die im Bezirk Finnmark früher auch weiter im Westen - an der Küste des Nördlichen Eismeers gesprochenen ursprünglich ostlappischen Dialekte zusammengefasst, die durch die an die Küste kommende gebirgslappische Siedlungsbewegung allmählich in einem Ausmass westlappische Züge angenommen haben, dass sich die ursprüngliche Zugehörigkeit zum Ostlappischen nur noch in einigen rudimentartigen Besonderheiten ausdrückt. Die Verfasserin begründet ihre Auswahl des Forschungsgegenstandes damit, dass auf diese Weise sowohl die ost- als auch die westlappische Sprachform behandelt wird. Das Seelappische ist eine Art Verbindungsglied; hierdurch kommt ein geographisch fast einheitliches Gebiet zustande.

Das Forschungsmaterial stammt zum grössten 'T'eil aus der Literatur. Mehr oder minder spontane Volkssprache ist in folgenden Quellen enthalten: Qvigstad "Lappiske eventyr or sagn" und "Lappische Texte aus Kalfjord und Helgöy", Lagercrantz "Lappische Volksdichtung", Ravila "Ruijanlappalaisia kielennäytteitä Petsamosta ja Etelä-Varangista (Finnmarklappische Sprachproben aus Petsamo und Süd-Varanger)», inarilappische Artikel in der Zeitung "Sabmelaš", Koskimies und Itkonen "Inarinlappalaista kansantietoutta (Inarilappische Volksüberlieferung) sowie Anta Pirak "Jåhttee saamee viessoom». Einen anderen Charakter hat die inarilappische Übersetzung der Bibelgeschichte von Lauri Itkonen. Man kann geteilter Meinung darüber sein, inwieweit sich ein Übersetzungstext - noch dazu ein religiöser - als Untersuchungsmaterial eignet, wenn die Syntax eines echten Dialektes erforscht werden soll. Hier wird die Entscheidung der Verfas- 
serin beeinflusst worden sein von der geringen Anzahl vorhandener inarilappischer Textquellen. Neben den literarischen Quellen wurden auch Archivmaterial aus dem Bereich des inarilappischen Wörterbuches sowie inarilp. eigene Aufzeichnungen der Verfasserin aus dem Terrain verwendet.

Das Seelappische ist verglichen mit den beiden anderen untersuchten Dialekten in zwei Hinsichten relativ uneinheitlich. Zunächst einmal hat sich der Übergang zum Westlappischen unterschiedlich vollzogen, je nachdem, welches Gebirgslappisch in der Nachbarschaft gesprochen wurde. Zweitens kommt der Einfluss des Gebirgslappischen verschieden stark zum Ausdruck. Eine scharfe Grenze zwischen dem See- und Gebirgslappischen gibt es nicht, obwohl bestimmte, vor allem lautliche Kriterien normalerweise als Kennzeichen des Seelappischen angesehen werden. Es wäre gut gewesen, hätte die Verfasserin kurz die Grundsätze dargelegt, nach denen sie ihr seelappisches Material ausgewählt hat. Es erscheinen einige Beispielsätze im Buch, die deutliche gebirgslappische Besonderheiten enthalten, z.B. das Klusilwerden des $j$ (dud'djut, S. 33, bâddje-sam'melẩ̆ $\breve{z} \hat{a} k$, S. 37) und ein prothetischer Klusil vor einem Nasal (fabmo, S. 33, sudnji, S. 89, sodnost, S. 128). Diese Fälle sind jedoch zahlenmässig sehr gering und sie sind alle so geartet, dass sie in keiner Weise die Behandlung der Kasussyntax selbst beeinflussen.

Die Abhandlung ist klar gegliedert, allerdings überaus detailliert, was schon daraus hervorgeht, dass das Inhaltsverzeichnis sieben Seiten umfasst. Nach einer kurzen und komprimiert geschriebenen Einleitung (S. 12-17) geht die Verfasserin zur ausführlichen Behandlung der Verwendung der Kasus über, was den grössten Teil des Buches ausmacht (S. 18-142). Im gesamten Untersuchungsgebiet begegnen die Kasus Nominativ, Akkusativ, Genitiv, Essiv, Komitativ, Abessiv und Illativ. Im Lulelappischen begegnet ausserdem der Inessiv und Elativ, denen im Inari- und Seelappischen ein Kasus entspricht, der Lokativ nämlich. Im Inarilappischen gehört noch der Partitiv zum Nominalparadigma. Die Kasus werden in der oben genannten Reihenfolge behandelt. Da im Inari- und Seelappischen Akkusativ und Genitiv Singular mit Ausnahme einiger Pronomina und dialektal im Seelappischen auch der Akkusativ und der Genitiv Plural zusammengefallen sind, werden die Fälle, bei denen sich nicht entscheiden lässt, ob es sich um einen Akkusativ oder Genitiv handelt, in einem eigenen Kapitel unter dem Namen Genitiv-Akkusativ behandelt. Falls die Funktionen des Lokativs im Inari- und Seelappischen denen des Inessivs oder Elativs im Lulelappischen entsprechen, werden sie im Zusammenhang mit dem Inessiv und Elativ abge- 
handelt. Jene Funktionen des Lokativs, die keine Entsprechung im Lulelappischen haben, sind in einem Kapitel "Andere Funktionen des Lokativs" zusammengefasst. Auf die Analyse der Verwendung der Kasus folgen Kapitel mit den Überschriften "Die Kasus als Systemganzes" (S. 143-154), "Die sog. schwache Flexion" (S. 155-160) und "Unterschiede der inari-, see- und lulelappischen Kasussysteme» (S. 161--164). Den Quellen- und Abkürzungsverzeichnissen folgt noch ein Referat in deutscher Sprache (S. 168-180).

Das theoretische Gerüst der Arbeit hat Frau Bartens selbst gebaut, auch wenn sie sich in einigen Punkten auf aus- und inländische Syntaktiker stützt wie John Lyons, E. Křižkova, Jaan Kaplinski und Paavo Siro. Die Funktionen der Kasus werden eingeteilt in zentrale und periphere. Die zentralen Satzglieder sind vom Standpunkt des Satzinhaltes notwendig, die peripheren dagegen variieren lediglich die grundlegenden Satztypen. Zu den zentralen Satzgliedern gehören das Subjekt, das Prädikat, das Prädikativum und die verschiedenen obligatorischen Ergänzungen beim Verb, wie das Objekt und die vom Verb verlangten Adverbialia. Periphere Satzglieder sind u.a. die fakultativen Komplemente, die Attribute des Verbs und das Agens. Unter dem Verbattribut versteht die Verfasserin ein solches Adverbiale beim Verb, das in einem Satz mit einem intransitiven Verb nicht zum Netzwerk von Subjekt und Prädikat und in einem Satz mit einem transitiven Verb nicht zu dem von Objekt und Prädikat gehört. Es handelt sich um einen neuen, von der Verfasserin entwickelten syntaktischen Begriff, durch den die Klassifizierung der verbalen Bestimmungen treffend präzisiert und ergänzt $\mathrm{zu}$ werden scheint. Die durch die generative Syntax gebotenen Möglichkeiten hat Frau Bartens nur wenig ausgenutzt. Vor allem die Beziehungen zwischen den fakultativen Komplementen des Verbs und den anderen Satzgliedern sowie das freie Attribut des Substantivs werden mitunter mit Hilfe einer Insertion veranschaulicht. Weiter hätte eine generative Behandlungsweise auch kaum gehen können, da der Forschungsgegenstand nicht die Muttersprache der Verfasserin ist und ihr keine eingeborenen Informanten zur Verfügung standen, deren syntaktische Intuitionen sie hätte erforschen können.

Der des Lappischen nicht näher kundige Leser mag eine Darstellung der Kasusmorphologie vermissen. Ein kurzer morphologischer Überblick wäre schon deshalb angebracht gewesen, weil die Arbeit ihrer zahlreichen geglückten Lösungen wegen offenbar nicht nur bei den Lappologen Interesse wecken und Aufmerksamkeit erregen wird. Am besten hätte eine solche İ́bersicht in die Einleitung gepasst. 
Die Darstellung der Verwendung der Kasus zeugt durchwer von geschultem syntaktischen Denken und sensiblem Satzgefühl. Die Konstruktionen und die Funktionen der darin befindlichen Kasus sind meist zutreffend ausgelegt. Die Regeln werden richtig und mit genügender Vorsicht vom Beispielmaterial abgeleitet. Nicht in allen Fällen liess das vorhandene Material schon eine endgültige Meinungsbildung zu; klugerweise hat die Verfasserin dann die Frage offengelassen. Direkte Fehler gibt es wenig. Hierher kann z.B. gerechnet werden, dass S. 1819 solche prädikatlosen Existentialsätze wie mut Sulgušjääyrist ciizzag 'aber im (See) Sulkusjärvi (sind sehr viele) Wasserinsekten' als Nominalsätze behandelt werden. An einigen Stellen hat die muttersprachliche Syntax der Verfasserin Schlussfolgerungen provoziert, die vom Lappischen her zweifelhaft wirken oder direkt irrtümlich sind. So wird S. 26 folgende Regel aufgestellt: "Wenn das Verb (I) lede lautet, ist das Verhältnis zwischen Subjekt und Prädikativum total oder partiell; wenn ein anderes Verb als lede verwendet wird, ist das Verhältnis ein totales." Die Begriffe Totalität und Partialität stammen aus der finnischen Syntax. Im Lappischen haben sie keine formale Entsprechung. Zu dieser unnötigen Analyse wurde die Verfasserin dadurch verleitet, dass in den finnischen Übersetzungen einiger Beispielsätze einem lappischen Prädikativum in Nominativform im Finnischen die Partitivform entspricht, z.B. lpI aainas lijjii mäáliih 'sondern die waren welche aus der Unterwelt' (vgl. fi. vaan ne olivat manalaisia), täat piipo lii munjin eeiji ärbi 'diese Pfeife ist mir ein Erbe vom Vater, d.h. habe ich vom Vater geerbt' (vgl. fi. tämä piippu on minulle isän perintöä).

Die Ausführungen von Frau Bartens sind im allgemeinen glaubhaft. In vielen Fällen bringen sie etwas entscheidend Neues, das auch ausserhalb des erforschten Dialektgebietes gilt. So trifft z.B. die Beobachtung (S. 74-75), eine solche Dual + Komitativ-Konstruktion wie muoj kilguinan 'ich und meine Frau' (wörtlich 'wir zwei mit meiner Frau') begegne im Inari-, See- und Lulelappischen fast ausschliesslich nur als Subjekt, offenbar für das gesamte Norwegischlappisch zu. Die früheren Grammatiken (Nielsen, Wiklund) erwähnen diese Einschränkung nicht.

Einigen Auslegungen kann man sich nicht vorbehaltlos anschliessen, obwohl man sie auch nicht immer unbedingt als falsch bezeichnen kann. In anderen Fällen blieb die Behandlung oberflächlich. Das Material hätte eine tiefergehende Erörterung und reifere Schlussfolgerungen gestattet. Im folgenden beschäftige ich mich eingehend mit einigen solchen Punkten, wo eine von der Ansicht der Verfasserin abweichende Ansicht 
besser motivierbar sein kann oder wo man eine genauere Untersuchung erwartet hätte.

Die Verfasserin behandelt S. 23 lulelappische Passivsätze, die zwei Nominative haben, z.B. åa $i v e$ e vuolaas $j$ ai piejahtuvvaa $j u$ ok a piv val 'als Kopfunterlage wird etwas Warmes hingelegt', liaarvoohtij muorra-piehkèr, m $i$ lij vuolahtum a $a i^{\prime} v$ e e '(er) bekleidete ein Holzstück, (an) dem ein Kopf geschnitzt worden war'. Sie stellt fest, der eine Nominativ würde im entsprechenden Aktivsatz eine Essiv- oder Lokalkasus-Bestimmung vertreten, erörtert jedoch nicht, ob solche Passivsätze im Transformationsverhältnis $\mathrm{zu}$ solchen Aktivsätzen stehen könnten, wo neben dem Objekt ein akkusativisches Objektprädikativum erscheint, z.B. te t av a le$m a v$ kååruh låau'taka padjee-rapte mil'te $s \ddot{a} m m a a k u h$ $k$ ì $v$ 'den Rauchfangteil nähen sie am Oberrand der Zeltbedeckung ebenso lang (= in gleicher Länge) an' (S. 43), und besonders zu gerade für das Lulelappische typischen Aktivsätzen mit zwei Objekten, wo das eine Objekt eine Gesamtheit und das andere einen Teil davon ausdrückt, z.B. pier'na aal'kij

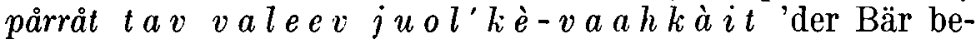
gann die Waden jenes schnellen Mannes zu beissen' (S. 32).

Die Verbindung des Akkusativobjekts mit Nominalformen des Verbs ist ausführlich und sichtlich zuverlässig S. 32-42 behandelt worden. S. 35 heisst es, "das Akkusativobjekt verbindet sich mit der Elativ-, Inessiv- oder Illativform der Aktion, die selbst als Adverbiale des Prädikatsverbs (oder der zum Prädikat gehörenden Nominalform) auftritt». Für ein an den Illativ der Aktion tretendes Objekt hat Raija Bartens nur ein Beispiel, lpI vajk Immeel lišij tooin naaliin Sammlažvuorras puäradasan $j \dot{a} \dot{a} \dot{a} r m u$ uuccaman kihtalam 'obwohl Gott auf diese Weise den alten $S$. zur Busse und zur Gnadensuche angeregt hätte'. Auch hier wird es sich kaum um ein Objekt handeln, sondern eher um ein an den Illativ der Aktion tretendes Genitivattribut. Für ein mit dem Illativ der Aktion verbundenes Objekt liegt kein sicheres Beispiel vor, während ein Genitivattribut der Aktion ziemlich üblich ist. Auch der Gen.-Akk. Sg. im Satz lpI koolgah išedid $k \dot{a} r b \dot{a}$ sačamist jäauran 'du sollst dabei helfen, das Boot ins Wasser zu stossen (wörtl. du sollst beim Boot-Stossen in den See helfen)' (a.a.0.) kann ebensogut ein Genitivattribut wie ein Objekt sein, denn im Prinzip können beide Satzglieder als Lokativbestimmung der Aktion in Frage kommen, vgl. z.B. lpI vi ermij juonastmist 'vom unter-Eis-Auslegen der Netze' (S. 53), wo die Bestimmung eindeutig der Gen. Pl. ist.

Unter Vorbehalt reiht Raija Bartens den Beispielen mit Akkusativformen die seelappischen Fälle gulai bieggâa šuvvâm 
'hörte den Wind rauschen' und de gullen rangu čuör'rom sie hörten das Meergespenst rufen' (S. 39) ein. Sie hält es für möglich, dass šuvvâm und čuör' com aus dem stellenweise im Norwegischlappischen auftretenden, ant -me, mě endenden Gerundium II durch Apokope entstanden wären. Diese Möglichkeit braucht jedoch kaum berücksichtigt zu werden, demn im Veranger Seelappisch, woher der obige Beleg stammt, erscheint das Gerundium II konsequent mit der Endung -men (z.B. S. 38 und 39 sukkâmen 'beim Rudern', guk'kâmen 'beim Kukkuckrufen'), während das Gerundium II in der Mundart von Kalfjord-Helgöy, die das letztere Beispiel vertritt, auf $-n$ ausgeht, mannan 'im Gehen', boatten 'im Kommen' usw. (Qvigstad, Lappische Texte aus Kalfjord und Helgöy S. 24-25, nach E. Itkonen ${ }^{*}$ boattemen $>{ }^{*}$ boattem ${ }^{E}{ }_{n}>{ }^{*}$ boattemn $>$ boatten, s. MSFOu XCVIII S. 280). Somit sind die von Raija Bartens erwähnten seelp. Formen als Akkusative der Aktion 711 betrachten.

In Konstruktionen wie der oben erwähnten kann auch das Part. Perf. auftreten, wie S. 40 festgestellt wird, z.B. lpI oajna kyehti looza lappum 'sieht zwei Nägel verschwunden (d.h. dass zwei Nägel verschwunden sind)'. Nach Bartens kann eine solche Konstruktion in einen derartigen Nebensatz verwandelt werden, dessen Prädikatsverb im Perfekt steht. Das ist jedoch nur dann der Fall, wenn das Prädikat des Hauptsatzes im Präsens steht, wie im oben zitierten Beispiel. Wenn das Prädikat des Hauptsatzes im Präteritum steht, erscheint das der Nominalform entsprechende Prädikat des Nebensatzes im Plusquamperfekt. So lässt sich das von Frau Bartens angeführte Beispiel lpS oini manas jammam nu fâstet '(er) sah sein Kind auf so schreckliche Weise gestorben' ableiten aus dem Satzgefüge oini, âtte su manna lcei jammam nu fâstet '(er) sah, dass sein Kind auf so schreckliche Weise gestorben war'. Wie Schlachter nachgewiesen hat (Symposion über Syntax der uralischen Sprachen S. 190-191), wird gerade in solchen Fällen das Plusquamperfekt verwendet, zum Ausdruck der »Vorzeitigkeit in der Vergangenheity also.

Für die Genitivbestimmung des Adjektivs liegt nur ein Beleg vor: lpI mon nommsaž almaj 'ein wie heissender Mann (wörtl. welches [Namens] namiger Mann)' (S. 52). Es dürfte mehr diesbezügliches Material geben, denn solche Ausdrücke wie IpN $d \bar{u}$ lāgan 'wie du geartet', $d \bar{u}$ lakkasâs id., ol'bmu habmasâš 'menschlich aussehend', ovta-âllusẩ 'gleich hoch', guovtěgerdaš 'doppelt', àednâm-vuolaš 'unterirdisch' usw. (s. Nielsen, Lærebok i lappisk I S. 225, 227, 311-312) dürften relativ häufig sein. Konstruktionen diesen Typs enthalten sehr viel Komposita. Dies erklärt, warum Raija Bartens nicht mehr 
Belege bringt, denn sie hat die syntaktischen Beziehungen zwischen den Teilen von Komposita gar nicht in ihre Untersuchung einbezogen. Damit man ein Gesamtbild bekommt von der Verwendung des Genitivs als Adjektivbestimmung wäre es empfehlenswert gewesen, dass die Verfasserin in diesem Punkt von ihrem an sich einleuchtenden Grundsatz abgewichen wäre und auch die zusammengesetzten Adjektive mit einem Genitiv als erstem Glied behandelt hätte. Dieses Verfahren wäre auch dadurch gerechtfertigt worden, dass die Grenze zwischen Zusammensetzung und Wortgefüge in diesen Fällen nicht ganz selbstverständlich ist. Da es sich um schriftlich aufgezeichnete Sprachproben handelt, gibt es nicht einmal Garantien dafür, dass die Auslegung des Aufzeichnenden immer.unbedingt richtig ist.

Von den Funktionen des Genitivs und Akkusativs ist nur eine geblieben, die nicht im Zusammenhang mit dem Genitir oder Akkusativ hat behandelt werden können, sondern in einem eigenen Kapitel "Genitiv-Akkusativ» (S. 57-58) abgehandelt wurde. Im Seelappischen sieht das als Prädikativum stehende Adjektir dann wie ein Genitiv-Akkusativ aus, wenn das Subjekt pluralisch oder dualisch ist, z.B. dâk lejije nu $h i r$ $r \hat{a} s$ 'die waren so scheu'. In den Ostdialekten des Seelappischen betrifft dies nur im Stamm ungleichsilbige Adjektive, vor allem solche, die auf $-s$ enden. In den Westdialekten gibt es solche Beschränkungen nicht. Nachdem die Verfasserin dargrestellt hat, wie frühere Forscher diese Formen deuteten, bringt sie auch ihre eigene Erklärung. Danach wäre der gesamte T'ypus von den $s$-Adjektiven ausgegangen, die im Seelappischen als Prädikative ursprünglich unflektierbar gewesen wären, wie sie es im Inarilappischen noch heute sind. Der Druck der übrigen Adjektivtypen hätte verursacht, dass das auf ein phuralisches Subjekt hinweisende Prädikativum eine andere Form annahm als das auf ein singularisches Subjekt hinweisende, wobei nur die Hauptsache war, dass sich die Form deutlich vom Nominativ Singular unterschied. Eine solche Entwicklung dürfte im Prinzip nicht unmöglich sein. Es erhebt sich jedoch die Frage, auf welches Muster sich die neue pluralische Prädikativform stützte. Falls es kein Vorbild gibt, bleibt die Erklärung von Frau Bartens unvollständig und in starkem Masse unwahrscheinlich. Glaubhafter wirkt da die Auslegung von Bergsland (Studia Septentrionalia V S. 62-63), die von der Verfasserin sichtlich übersehen worden ist. Nach Bergsland setzen diese dem Gen. Sg. entsprechenden Prädikativformen wie auch z.B. einige in Verbindung mit Zahlwörtern auftretende ähnliche Formen den urlappischen Nom. Pl. regelmässig fort. Das Pluralzeichen -l; wäre also lautgesetzlich ge- 
schwunden, später jedoch in der normalen Nominalflexion nach dem Muster der einsilbigen Pronomina wieder eingeführt worden. Die regelgemässe Schwundvertretung hätte sich nur in solchen Konstruktionen erhalten können, wo ein Pronomen nicht in Frage kommen kann, wie in Prädikativa und in Verbindung mit Zahlwörtern. (Zu Zahlwortkonstruktionen vgl. E. Itkonen FUF XL S. 324.) Im Lichte der von Bergsland gegebenen Erklärung ist es durchaus verständlich, dass ein dem Gen. Sg. ähnliches pluralisches Prädikativum in den seelappischen Ostdialekten eben von Adjektiven mit ungleichsilbigem Stamm gebraucht wird. Gerade in diesem Stammtyp ist z.B. nach dem Schwund der Endung von Gen. und Akk. Sg. auch der Auslautvokal des Stammes geschwunden. Das geschah natürlich auch, wenn das Pluralzeichen $-k$ geschwunden ist. Die Wiedereinführung eines $-k$ in einem zweisilbigen, konsonantisch auslautenden, eine ursprüngliche Pluralform vertretenden Stamm (z.B. gar'vas 'fertige') geht natürlich nicht so leicht wie in der entsprechenden Form von im Stamm gleichsilbigen Adjektiven (z.B. buore 'gute'), wo der Auslautvokal stets erhalten ist.

Unter den Belegen für den habitiven Inessiv (- Hım) gibt es einige, wo der Inessiv vielleicht eher lokal als habitiv ist: IpI $t \dot{a} \dot{a} n l i \grave{s} s \dot{a} \dot{a} s t$ lii šieu $\dot{a} v j u$ 'diese Sense hat eine gute Klinge', tan $l$ ĩ $i n$ es $t$ lii mučis ivne 'dieses Tuch hat eine schöne Farbe', lpS mu bursâst $i$ lak obbâ bon'nege 'in meiner Börse ist gar kein Boden' (S. 105-106). Doch wird man die Auslegung der Verfasserin akzeptieren können, falls die Habitivität weit gefasst wird.

Auf den Seiten 106-107 kommt die Inessivrektion des Verbs zur Sprache, von der festgestellt wird, dass sie selten ist. Es wird kein Zufall sein, dass alle diesbezüglichen Beispiele aus dem Lulelappischen stammen und dass die in Frage kommenden Verben 'hineinpassen' und 'leser' bedeuten. Die entsprechenden schwedischen Verben erhalten die Präposition $i$, so dass hier offenbar ein Einfluss der schwedischen Syntax auf das Lulelappische vorliegt.

Im Abschnitt über den Inessiv als Kasus der Partikelbestimmung (S. 113) hat sich ein falsches Beispiel eingeschlichen: lpS guovte gerde vâkkost 'zwei Mal in der Woche'. Der Inessir ist hier nicht die Bestimmung der Partikel, sondern des Gen.Akk.-förmigen Substantivs.

Es kann gefragt werden, ob es zweckmässig ist, die Elativform in solchen Fällen wie lpS geeč čâlâsti mallasest '(er) kostete etwas Suppe (eigtl. ron der Suppe)' (S. 114) als Objekt und in solchen Sätzen wie lpL kaaihtsa-miel'kès lä hui valljee 'Ziegenmilch (eigtl. von der Ziegenmilch) gibt es sehr viel' (S. 118) 
als Subjekt zu erklären. Die Einführung von Elativsubjekt und -objekt in die lappische Syntax zerstört das sonst so einfache und klare Subjekt- und Objektregelsystem, wonach der Kasus des Subjekts der Nominativ ist und der des Objekts der Akkusativ. Obgleich auch Begründungen vorhanden sind für die Lösung von Frau Bartens, ist damit doch wohl ein zu grosses Opfer verbunden. Die stärksten Argumente liegen für ein Elativobjekt vor, denn die objektartige Verwendung des Elativs ist im Lappischen relativ allgemein und auch systematisch. Die zwei Beispielsätze aber, in denen die Verfasserin ein Elativsubjekt annimmt, lassen sich auch auf eine andere und zur Gesamtheit besser passende Weise gliedern. Historisch betrachtet ist es interessant, dass weiterhin, obwohl der im Urlappischen offenbar herrschende Unterschied zwischen totalem und partiellem Objekt in den lappischen Dialekten geschwunden ist, ein gewisser Trend zum Ausdruck der Partialität vorhanden ist; da der alte Kasus des partiellen Objekts, der Partitiv, diese seine Funktion eingebüsst hat, wird der Elativ zum Indikator der Partialität. Die Geschichte wiederholt sich insofern, als es wieder ein Trennungskasus ist, durch den das partielle Objekt ausgedrückt wird.

Es war eine gute Entscheidung, im Kapitel "Andere Funktionen des Lokativs" solche inari- und seelappischen Lokativfälle unterzubringen, die keine Entsprechung im Lulelappischen haben. An manchen Punkten scheint die Verfasserin fast zu vorsichtig gewesen zu sein und Fälle in dieses Kapitel hereingenommen zu haben, die durchaus entweder mit dem Elativ oder mit dem Inessiv hätten in Verbindung gebracht werden können. So dürften die S. 137 im Abschnitt 11.2.1.1. (Der Lokativ als Kasus des Verbalattributs) im letzten Absatz behandelten Lokative des Grundes und der Ursache ohne weiteres als Elative gedentet werden können, z.B. lpI taggaar

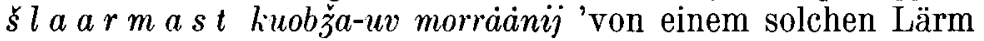
wachte auch der Bär auf'. Der mit dem Lokativ der Ursache eng verwandte Lokativ des Hinderungsgrundes ist denn auch aufgrund der lulelappisehen Beispiele (kutih smaavva $m a a_{-}$ $n \dot{a} i s$ päässih vuol'kèt 'die kleine Kinder loslassen' (eigtl. 'die von kleinen Kindern losgehen') beim Elativ untergebracht (S. 131, Punkt 10.2.2.2.). Ausserdem erwähnt K. B. Wiklund in seinem lulelappischen Lehrbuch (Lärobok i lapska språket, 2. Aufl., S. 108) ausdrücklich, dass der Elativ den Grund angibt, weshalb etwas geschieht. Von seinen zwei Beispielen gehört das eine, $\bar{\imath}$ sitāh vuöleket nahkiāris '(er) wollte nicht vom Schlaf (d.h. noch schläfrig) losgehen', nach der Gliederung von Raija Bartens zum Elativ, der den Hinderungsgrund ausdrückt, während es sich im zweiten, sinn jāmi tat hāvēs 'er starb 
an dieser Wunde' gerade um einen solchen Elativ des Grundes handelt, den Raija Bartens mit dem Lokativ verbindet. Es dürfte ein blosser Zufall sein, dass ein solcher Elativ nicht im lulelappischen Material von ihr enthalten war.

Der Hauptabschnitt "Die Kasus als Systemganzes" ist durchweg sachlich und klar geschrieben. Anfangs wird darin untersucht, welche Kasus in welcher syntaktischen Funktion erscheinen. Auf Schlachter gestützt teilt die Verfasserin den Kasusgebrauch in ein System bildende Systemfälle und sich aufgrund des Kontextes erklärende Regelfälle, in definierbare Ausnahmefälle, die kein System bilden. Diese Teilung erweist sich als nützlich, denn gerade im Lichte der Systemfälle wird die Aufgabenteilung der Kasus klar: als zentrale Satzglieder treten der Nominativ, Akkusativ, Essiv, Illativ und Inessiv auf, als periphere wiederum der Genitiv, Komitativ, Abessiv, Elativ und Partitiv. Am Ende des Abschnittes werden die Kasusfunktionen noch von dem Standpunkt betrachtet, ob sie von yrammatischen oder lexikalischen Gesichtspunkten her bestimmt werden.

In einem gesonderten Hauptabschnitt wird S. 155-160 die sog. schwache Flexion behandelt, worunter man versteht, dass einige Pronomina, Zahlwörter, Adjektive und lokale Verhältnisse ausdrückende Nomina als Adjektivattribute kongruieren, anders als die lappischen Adjektive im allgemeinen. Diese Kongruenz ist jedoch nicht vollständig, sondern in der schwachen Flexion gibt es weniger Attributskasus als im eigentlichen substantivischen Paradigma. Somit begegnet z.B. der Gen.Akk. Sg. des Attributs im Inari- und Seelappischen ausser vor einem Bezugswort in Gen.-Akk.-Form auch vor einem solchen in Ill.- und Lok.-Form, z.B. Nom. Sg. tait ihe 'dieses Jahr', Gen.-Akk. täan iyve, Ill. täan ihän, Lok. täàn ivveest usw. Die schwache Flexion von der übrigen Kasussyntax getrennt zu behandeln ist motiviert und bringt u.a. den Vorteil, dass sich der Leser ein gutes Allgemeinbild machen kann von der Erscheinung, ohne die hierher gehörigen Fälle an verschiedenen Stellen im Buche suchen zu müssen.

Im Kapitel "Unterschiede der Kasussysteme im Inari-, Seeund Lulelappischen" sind jene Züge der Kasussyntax versammelt, in denen die Verfasserin aufgrund ihrer Untersuchung interdialektale Unterschiede festgestellt hat. Da das Forschungsmaterial begrenzt war und zusätzliches Material das Bild in einigen Einzelheiten verändern könnte, konzentrierte sich die Verfasserin nur auf solche Unterschiede, wo der Anteil des Zufalls nicht berücksichtigt werden braucht. An den Differenzen zeigen sich manche interessante dialektgeographische Besonderheiten, aus denen auch sprachgeschichtliche Schluss- 
folgerungen zu ziehen wären. Das hat Raija Bartens jedoch nicht getan, da ihre Arbeitsweise auch sonst durchgehend synchron ist. Der Leser vermisst jedoch einen etwas weitergehenden Vergleich. Interessante Resultate hätte z.B. ein Vergleich der syntaktischen Cnterschiede mit Differenzen ergeben, die in anderen Bereichen der Grammatik auftreten. Es sei nur darauf hingewiesen, dass sich das Lulelappische in del' Kasussyntax deutlich rom Inari- und Seelappischen unterscheidet, die ihrerseits einander sehr nahe stehen; hinsichtlich der Phonologie und der Morphophonologie verläuft die schärfste Grenze zwischen dem Inari- und Seelappischen oder allgemeiner zwischen dem Inari- und Norwegischlappischen, während wiederum zwischen dem See- und Lulelappischen kein so grosser Unterschied besteht. Ein ergiebiges Forschungsobjekt wäre ferner die Frage gewesen, in welchem Umfang die Sprachen der Mehrheiten, Finnisch, Norwegisch und Schwedisch, auf die Syntax eines jeden Dialektes eingewirkt haben und in welchem Ausmass sich die Unterschiede durch fremden Einfluss erklären bzw. inwieweit sie auf die historische Einteilung Ostlappisch - Westlappisch zurückgehen.

Im Quellenverzeichnis fehlt die schwedische U̇bersetzung von Anta Piraks Buch Jåhttee siamee viessoom, die getrennt i.J. 1933 unter dem Titel En nomad och hans liv erschien. Aus dieser Übersetzung wird S. 21 zitiert. Auch John Lyons, Introduction to Theoretical Linguistics ist nicht im Quellenverzeichnis aufgenommen, vielleicht weil die Grundsätze von Lyons nicht direkt sondern aus der Arbeit von Valma YliVakkuri übernommen wurden.

In der deutschen Zusammenfassung liegt das Hauptgewicht auf der Darstellung des Abschnittes "Die Kasus als Systemganzes». Dies ist durchaus angebracht, denn in diesem Abschnitt sind die wichtigsten Ergebnisse der Arbeit enthalten.

Vom formalen Standpunkt aus ist die Untersuchung von Raija Bartens lobenswert. Sie drückt sich durchweg präzis und geschlossen aus. Die Gliederung ist zweckmässig klar. Die Korrektur ist sorgfältig gelesen worden, so dass es sehr wenig eigentliche Druckfehler gibt. Kleine Flüchtigkeitsfehler lassen sich hier und da entdecken, doch ist auch ihre Anzahl so niedrig, dass sie den positiven Gesamteindruck, den auch die äussere Form des Buches gibt, nicht beeinträchtigen.

Die Arbeit von Raija Bartens ist die erste systematische Darstellung der lappischen Kasussyntax. Die darin getroffenen selbständigen und die Forschung fördernden syntaktischen Entscheidungen lassen sich weitgehend auch für die sonstige syntaktische Forschung verwenden. Weil die Untersuchung drei verschiedene Dialekte behandelt, ist sie auch als Quelle 
für die historisch-vergleichende Syntax geeignet. Die Abhandlung lässt auch als solche einige sprachgeschichtliche Schlussfolgerungen zu. So reiht sich diese verdienstvolle Arbeit vollendet in die Serie jener Monographien ein, die in der letzten Zeit über die Syntax der finnisch-ugrischen Sprachen geschrieben worden sind.

Mikko Korhonen

\section{Lehrbücher des Ungarischen}

Is'Tván NyIRkos, Nykyunkarin oppikirja (Lehrbuch der ungar. Gegenwartssprache). Tietolipas 71. SKS. Helsinki 1972. $172 \mathrm{~S}$.

István NriRkos, Unkarilais-suomalainen sanakirja / Magyarfinn szótár (Ungar.-finn. Wörterbuch). SKS. Helsinki 1969. $392 \mathrm{~S}$.

Dr. István Nyirkos war in den Jahren 1962-67 an der Universität Helsinki als Lektor für Ungarisch tätig. Damals wurden von den Studenten des Ungarischen das kleine »Unkarin oppikirja» (Ungar. Lehrbuch; erschienen 1961) von Lavotha -Tervonen und eine von György Lakó redigierte Chrestomathie benützt. I.J. 1965 publizierte Nyirkos eine neue Chrestomathie ("Unkarin lukemisto sanastoineen" [Ungar. Lesebuch mit Wörterverzeichnis]). Nach Abschluss seiner langen Lektorentätigkeit, während der er viele und interessierte Schüler hatte, verfasste er für die finnischen Interessenten des Ungarischen noch ein "Nykyunkarin oppikirja" (Lehrbuch der ungar. Gegenwartssprache, erschienen 1972 als Teil 71 der Tietolipas-Reihe der Finn. Literaturgesellschaft). Das Buch ist nicht nur für den Universitätsunterricht gemeint sondern auch für Autodidakten, z.B. für Touristen; die Themen der Lesestücke und der Wortschatz sind vom Verfasser so gewählt, dass der tägliche Sprachgebrauch des Ungarischen darin geboten wird.

Das Lehrbuch ist in 22 Lesestücke eingeteilt. Dazu gehören jeweils der Wortschatz, ausführliche grammatische Kommentare und zahlreiche Übungen. Nach den Lesestücken folgt ein alphabetisches ungarisch-finnisches Wörterverzeichnis und eine finnisch-ungarische Wörterliste zur Erleichterung der Übungsaufgaben. Im Teil "Grammatische Schwerpunkte" am Schluss des Buches sind einige Tabellen zur Flexion der Nomina und Verba versammelt.

Am Anfang des Buches findet sich im Einleitungsteil ein îherblick über die Orthographie und Aussprache des Unga- 